\section{Recent Advances in EMG Pattern Recognition for Prosthetic Control}

\author{
Ameri A. ${ }^{\oplus}$, Ghorbani M. ${ }^{{ }^{*} \odot}$
}

$\mathrm{L}$ imb loss results in significant debilitation and reduces the quality of life of the affected individuals [1]. To restore the lost limb's function, myoelectric systems have been widely used in powered prostheses [2]. With this approach, the motor intent is estimated from the electromyogram (EMG) signals recorded by electrodes which are placed on the skin surface above the residual muscles [1]. The principle of commercial myoelectric schemes has not changed in several decades, and is referred to as conventional control [2]. This technique uses a measure of amplitude (such as mean absolute value over a time window) of the EMG signals recorded by electrodes placed at two control sites, preferably over a pair of antagonist muscles of the residual limb, to control a single motion i.e. degree of freedom (DoF), for example hand opening closing [2]. To change the DoF, a mode switch is conducted by muscle co-contraction or a hardware switch [2]. The mode switch, however, results in an unnatural control of multiple DoFs [2].

To overcome this challenge, a significant body of research has been conducted on pattern recognition techniques [3]. With this approach, a classifier is trained to discriminate between different DoFs, using patterns from multi-channel EMG input data. Promising results have been achieved in the literature for classification of several DoFs [2]. Since activities of daily living include simultaneous movements of multiple DoFs, combined motions must be also included as separate classes, and they have to be conducted in the training set [4]. The limitation of this approach, however, is that it does not allow the DoFs in combined motions to have different magnitudes. As a solution to this problem, regression-based systems have been proposed [5, 6], where a regressor is trained to estimate each DoF, using data from single and combined motions. This strategy provides independent simultaneous control, because it does not limit the DoFs to have the same amplitude. Classification and regression based systems are the two categories of pattern recognition methods.

Due to the high dimensionality of EMG signals, the EMG instantaneous values are not directly used as the inputs to classifiers/regressors [1]. Instead, a set of features is extracted from a time window (100-200 ms) of EMG signals [7]. Feature engineering is the process of design and extraction of features with the highest amount of useful information to maximize the classification/regression accuracy [8] Among various EMG features proposed in the literature, the Time Domain (TD) set [9] is the most popular set and includes mean absolute value, waveform length, zero-crossings, and slope sign changes.

The past few years have seen the advent of deep learning-based myoelectric control $[4,10]$. Deep learning can perform classification/regression tasks directly from high-dimensional raw data, without feature engineering [8]. Convolutional neural network (CNN) [11] is one of the most widely
${ }^{1} \mathrm{PhD}$, Department of

Biomedical Engineering and Medical Physics,

School of Medicine, Shahid Beheshti University of Medical Sciences,

Tehran, Iran

*Corresponding author: M. Ghorbani

Department of Bio-

medical Engineering and Medical Physics, School of Medicine, Shahid Beheshti University of Medical Sciences, Tehran, Iran

E-mail: mhdghorbani@ gmail.com

Received: 23 February 2020 Accepted: 3 March 2020 
Ameri A. and Ghorbani M.

used deep learning frameworks. The successive convolution layers of CNNs can learn useful features from the EMG data to estimate the motor intent [4]. As the outcomes of the previous studies [4, 10] confirm, CNNs outperform classical models such as support vector machines (SVMs) with engineered feature sets.

EMG pattern recognition schemes have yet to be deployed in commercial prostheses. The major challenge is performance degradation due to disturbances such as electrode shift, skin impedance change, muscle size variations, and learning effect [2]. Recent studies (e.g. [12, 13]) have proposed methods to improve the robustness of EMG pattern recognition to such disturbances. These methods as well as new deep learning schemes that eliminate feature engineering, may pave the way for commercial implementation of myoelectric pattern recognition prostheses. Moreover, independent simultaneous control can be achieved by using regression deep learning models. These promising methods have the potential to significantly outperform existing commercial systems. Consequently, the missing functions in people with limb loss can be restored more efficiently by delivering a more natural and intuitive control.

\section{Conflict of Interest}

\section{None}

\section{References}

1. Farina D, Aszmann 0 . Bionic limbs: clinical reality and academic promises. Science translational medicine. 2014;6(257):257ps12. doi: 10.1126/scitranslmed.3010453.

2. Scheme E, Englehart K. Electromyogram pattern recognition for control of powered upper-limb prostheses: state of the art and challenges for clinical use. Journal of Rehabilitation Research \& Development. 2011;48(6):643-59. doi: 10.1682/ jrrd.2010.09.0177. PMID: 21938652.

3. Jiang N, Dosen S, Muller K-R, Farina D. Myoelectric control of artificial limbs-is there a need to change focus? [In the spotlight]. IEEE Signal Processing Magazine. 2012;29(5):150-2. doi: 10.1109/MSP.2012.2203480.

4. Ameri A, Akhaee MA, Scheme E, Englehart K. Real-time, simultaneous myoelectric control using a convolutional neural network. PloS one. 2018;13(9):e0203835. doi: 10.1371/journal.pone.0203835. PMID: 30212573. Pubmed PMCID: PMC6136764.

5. Ameri A, Englehart KB, Parker PA, editors. A comparison between force and position control strategies in myoelectric prostheses. 2012 Annual International Conference of the IEEE Engineering in Medicine and Biology Society; San Diego, CA, USA: 2012. P. 1342-5. doi: 10.1109/EMBC.2012.6346186.

6. Ameri A, Scheme EJ, Englehart KB, Parker PA, editors. Bagged regression trees for simultaneous myoelectric force estimation. 2014 22nd Iranian Conference on Electrical Engineering (ICEE); Tehran, Iran: IEEE; 2014. P. 2000-3. doi: 10.1109/ IranianCEE.2014.6999871.

7. Englehart K, Hudgins B. A robust, real-time control scheme for multifunction myoelectric control. IEEE transactions on biomedical engineering. 2003;50(7):848-54. doi: 10.1109/TBME.2003.813539.

8. Bengio $Y$, Courville A, Vincent P. Representation learning: A review and new perspectives. IEEE transactions on pattern analysis and machine intelligence. 2013;35(8):1798-828. doi: 10.1109/TPAMI.2013.50.

9. Hudgins B, Parker P, Scott RN. A new strategy for multifunction myoelectric control. IEEE Transactions on Biomedical Engineering. 1993;40(1):82-94. doi: 10.1109/10.204774.

10. Atzori M, Cognolato M, Müller H. Deep learning with convolutional neural networks applied to electromyography data: A resource for the classification of movements for prosthetic hands. Frontiers in neurorobotics. 2016;10:9. doi: 10.3389/ fnbot.2016.00009.

11. Lecun $\mathrm{Y}$, Bottou L, Bengio Y, Haffner P. Gradient-based learning applied to document recognition. Proceedings of the IEEE. 1998;86(11):2278-324. doi: 10.1109/5.726791.

12. Prahm C, Paassen B, Schulz A, Hammer B, Aszmann 0 . Transfer learning for rapid re-calibration of a myoelectric prosthesis after electrode shift. Converging clinical and engineering research on neurorehabilitation II: Springer, Cham; 2017. p. 153-7. doi: 10.1007/978-3-319-46669-9_28.

13. Du Y, Jin W, Wei W, Hu Y, Geng W. Surface EMG-based inter-session gesture recognition enhanced by deep domain adaptation. Sensors. 2017;17(3):458. doi: 10.3390/s17030458. 